\title{
Soil pH and biome are both key determinants of soil archaeal community structure
}

\begin{abstract}
The mechanisms underlying community composition and diversity of soil archaea are poorly understood. We compared both total archaea and ammonia oxidizing archaea (AOA) using $16 \mathrm{~S}$ rRNA and amoA genes pyrosequencing respectively, in two different biomes: tropics (Malaysia), and temperate (Korea and Japan). Despite differences in characteristics of these biomes, we found that at the broad taxonomic level the dominant archaeal lineages are the same, except in certain instances (16S rRNA gene: group 1.1a Thaumarchaeota; amoA gene: Nitrososphaera and Nitrosotalea lineages). However, at the OTU level, both total archaea and AOA communities showed biome-specific patterns, indicating that at lower taxonomic levels biome differences are also important. In both biomes, total archaeal diversity showed a negative correlation with $\mathrm{pH}$, but a hump-shaped curve for AOA diversity, peaking at $\sim \mathrm{pH}$ 6.0. Within each biome, $\mathrm{pH}$ also emerged as the delimiting factor determining variation in community composition of both total archaea and AOA. Communities from each biome clustered separately, even at analogous pH levels. At the OTU level, certain shared OTUs did occur at approximately the same $\mathrm{pH}$ range in both biomes. We found that closely related OTUs of both total archaea and AOA respectively tended to co-occur, suggesting that in evolutionary terms these closely related lineages have conserved very similar ecological requirements. This predictability also strongly suggests that soil archaeal community assembly has strongly deterministic aspect. Overall, our findings emphasize that soil archaeal communities are to large extent predictable and structured by both biome and by soil chemical environment, especially $\mathrm{pH}$.
\end{abstract}

Keyword: Ammonia oxidizing archaea; Biome; Community assembly; Soil pH; 16S rRNA gene 